\title{
Ring-Like Structures Corresponding to MV-Algebras via Symmetric Difference*
}

\author{
By \\ Ivan Chajda and Helmut Länger \\ (Vorgelegt in der Sitzung der math.-nat. Klasse am 25. März 2004 \\ durch das w. M. Ludwig Reich)
}

\begin{abstract}
The well-known natural bijective correspondence between Boolean algebras and Boolean rings is generalized from Boolean algebras to MV-algebras. The ring-like structures arising this way correspond in a natural bijective manner to so-called strong De Morgan algebras.
\end{abstract}

AMS Subject Classification: 06D35, 06D30.

Key words: MV-algebra, pseudoring, De Morgan algebra, strong De Morgan algebra.

\section{Introduction}

The fundamental operations in logic are disjunction, conjunction and negation. Describing them algebraically leads to lattice-like structures with some sort of complementation. In the classical case one obtains Boolean algebras which correspond to Boolean rings in a natural bijective way. Ring addition and ring multiplication can be logically interpreted as exclusive disjunction and conjunction, respectively.

\footnotetext{
* Research supported by ÖAD, Cooperation between Austria and Czech Republic in Science and Technology, grant No. 2003/1.
} 
The natural bijective correspondence between Boolean algebras and Boolean rings was generalized from Boolean algebras to orthomodular lattices in [9] and [17], to ortholattices in [2], to bounded lattices with an involutory antiautomorphism in [10], to pseudocomplemented semilattices in [4], to generalized orthomodular lattices in [6] and to generalized ortholattices in [5]. In [3] the interplay between the structures introduced in [2] and [10] is investigated. The structures introduced in [10] were studied in more detail in [11]-[15].

The interplay between lattice distributivity on the one side and associativity of ring addition and ring distributivity on the other side is of importance when applying the considered structures in the foundations of axiomatic quantum mechanics. E.g., the fact that in the associativity law only four operation symbols are involved whereas the number of operation symbols occurring in the distributivity law is five has some physical meaning concerning the complexity of the corresponding physical experiments.

\section{MV-Algebras}

First we introduce the notion of an MV-algebra. These algebras provide an adequate semantics for the infinite-valued Lukasiewicz logic (cf. [1]).

Definition 2.1. An $M V$-algebra is an algebra $(A, \oplus, \neg, 0)$ of type $(2,1,0)$ satisfying the following axioms:

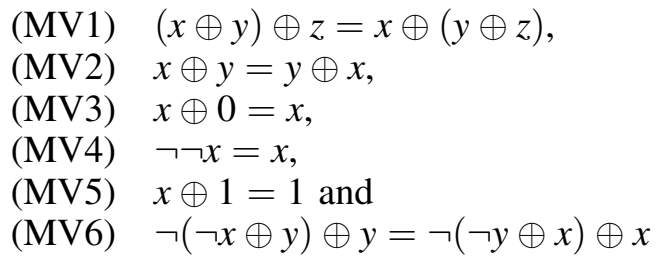

where 1 denotes the element $\neg 0$.

Example 2.1. $\left(B, \vee,{ }^{\prime}, 0\right)$, where $\left(B, \vee, \wedge,{ }^{\prime}, 0,1\right)$ is a Boolean algebra, is an MV-algebra.

Example 2.2. $([0,1], \oplus, \neg, 0)$, where $x \oplus y:=\min (x+y, 1)$ and $\neg x:=1-x$ for all $x, y \in[0,1]$, is an MV-algebra.

Lemma 2.1. In an $M V$-algebra $(A, \oplus, \neg, 0)$ it holds $x \oplus \neg x=1$ for all $x \in A$. 
Proof. For all $x \in A$ we have

$$
x \oplus \neg x=\neg x \oplus x=\neg(\neg 1 \oplus x) \oplus x=\neg(\neg x \oplus 1) \oplus 1=1 .
$$
by

In every MV-algebra $(A, \oplus, \neg, 0)$ a binary operation $\odot$ is defined

$$
x \odot y:=\neg(\neg x \oplus \neg y)
$$

for all $x, y \in A$. In $\left(B, \vee,{ }^{\prime}, 0\right)$, where $\left(B, \vee, \wedge,{ }^{\prime}, 0,1\right)$ is a Boolean algebra, $\odot=\wedge$ and in $([0,1], \oplus, \neg, 0)$ defined in Example 2.2, $x \odot y=\max (x+y-1,0)$ for all $x, y \in[0,1]$.

The following result is well-known (cf. [1]).

Proposition 2.1. If $(A, \oplus, \neg, 0)$ is an MV-algebra and one defines

$$
x \vee y:=\neg(\neg x \oplus y) \oplus y
$$

and

$$
x \wedge y:=\neg(\neg(x \oplus \neg y) \oplus \neg y)
$$

for all $x, y \in A$ then $(A, \vee, \wedge, 0,1)$ is a bounded distributive lattice the corresponding partial order of which is given by $x \leq y$ if and only if $\neg x \oplus y=1(x, y \in A)$.

For more results on MV-algebras we refer to the monographs [7] and [16].

\section{Pseudorings}

We now want to associate suitable ring-like structures to $\mathrm{MV}$-algebras. In this context it should be mentioned that connections between MV-algebras and semirings were investigated in [8].

Definition 3.1. A pseudoring is an algebra $(R,+, \cdot, 1)$ of type $(2,2,0)$ satisfying the following axioms:

$$
\begin{aligned}
& \text { (P1) }(x y) z=x(y z) \\
& \text { (P2) } x y=y x \\
& \text { (P3) } x 1=x \\
& \text { (P4) } 1+(1+x)=x \\
& \text { (P5) } x 0=0 \\
& \text { (P6) }(1+x(1+y))(1+y)=(1+y(1+x))(1+x) \text { and } \\
& \text { (P7) } 1+(1+x(1+y))(1+y(1+x))=x+y
\end{aligned}
$$

where 0 denotes the element $1+1$.

Remark 3.1. Axioms (P2) and (P7) imply the commutativity of + . 
The following theorem establishes a natural bijective correspondence between MV-algebras and pseudorings:

Theorem 3.1. If $A$ is a set then the formulas

$$
\begin{aligned}
& x+y:=\neg(x \oplus \neg y) \oplus \neg(\neg x \oplus y), \\
& x y:=\neg(\neg x \oplus \neg y)
\end{aligned}
$$

and

$$
\begin{aligned}
& x \oplus y:=1+(1+x)(1+y), \\
& \neg x:=1+x
\end{aligned}
$$

for all $x, y \in A$ induce mutually inverse bijections between the set of all $M V$-algebras on $A$ and the set of all pseudorings on $A$.

Remark 3.2. It holds $x+y=(x \odot \neg y) \oplus(\neg x \odot y)$ for all $x, y \in A$. Hence in $\left(B, \vee,{ }^{\prime}, 0\right)$, where $\left(B, \vee, \wedge,{ }^{\prime}, 0,1\right)$ is a Boolean algebra, + coincides with the symmetric difference and in $([0,1], \oplus, \neg, 0)$ defined in Example 2.2, + coincides with the distance of real numbers.

Proof of Theorem 3.1. If $(A, \oplus, \neg, 0)$ is an MV-algebra and $x+y:=$ $\neg(x \oplus \neg y) \oplus \neg(\neg x \oplus y)$ and $x y:=\neg(\neg x \oplus \neg y)$ for all $x, y \in A$ then

$$
\begin{aligned}
& 1+x=\neg(1 \oplus \neg x) \oplus \neg(\neg 1 \oplus x)=\neg x, \\
& 1+1=\neg 1=0, \\
& (x y) z=\neg(\neg \neg(\neg x \oplus \neg y) \oplus \neg z)=\neg((\neg x \oplus \neg y) \oplus \neg z) \\
& =\neg(\neg x \oplus(\neg y \oplus \neg z))=\neg(\neg x \oplus \neg \neg(\neg y \oplus \neg z))=x(y z), \\
& x y=\neg(\neg x \oplus \neg y)=\neg(\neg y \oplus \neg x)=y x \text {, } \\
& x 1=\neg(\neg x \oplus \neg 1)=x \text {, } \\
& 1+(1+x)=\neg \neg x=x \text {, } \\
& x 0=\neg(\neg x \oplus \neg 0)=0, \\
& (1+x(1+y))(1+y)=(\neg(x(\neg y)))(\neg y) \\
& =\neg(\neg \neg \neg(\neg x \oplus \neg \neg y) \oplus \neg \neg y) \\
& =\neg(\neg(\neg x \oplus y) \oplus y)=\neg(\neg(\neg y \oplus x) \oplus x) \\
& =\neg(\neg \neg \neg(\neg y \oplus \neg \neg x) \oplus \neg \neg x) \\
& =(\neg(y(\neg x)))(\neg x)=(1+y(1+x))(1+x), \\
& 1+(1+x(1+y))(1+y(1+x))=\neg((\neg(x(\neg y)))(\neg(y(\neg x)))) \\
& =\neg \neg(\neg \neg \neg(\neg x \oplus \neg \neg y) \oplus \neg \neg \neg(\neg y \oplus \neg \neg x)) \\
& =\neg(\neg x \oplus y) \oplus \neg(\neg y \oplus x)=x+y
\end{aligned}
$$


and

$$
1+(1+x)(1+y)=\neg((\neg x)(\neg y))=\neg \neg(\neg \neg x \oplus \neg \neg y)=x \oplus y
$$

for all $x, y, z \in A$.

If, conversely, $(A,+, \cdot, 1)$ is a pseudoring and $x \oplus y:=1+$ $(1+x)(1+y)$ and $\neg x:=1+x$ for all $x, y \in A$ then

$$
\begin{aligned}
& \neg 0=1+0=1+(1+1)=1 \text {, } \\
& (x \oplus y) \oplus z=1+(1+(1+(1+x)(1+y)))(1+z) \\
& =1+((1+x)(1+y))(1+z)=1+(1+x)((1+y)(1+z)) \\
& =1+(1+x)(1+(1+(1+y)(1+z)))=x \oplus(y \oplus z) \text {, } \\
& x \oplus y=1+(1+x)(1+y)=1+(1+y)(1+x)=y \oplus x \text {, } \\
& x \oplus 0=1+(1+x)(1+0)=1+(1+x) 1=1+(1+x)=x, \\
& \neg \neg x=1+(1+x)=x \text {, } \\
& x \oplus 1=1+(1+x)(1+(1+0))=1+(1+x) 0=1+0=1 \text {, } \\
& \neg(\neg x \oplus y) \oplus y=1+(1+(1+(1+(1+(1+x))(1+y))))(1+y) \\
& =1+(1+x(1+y))(1+y)=1+(1+y(1+x))(1+x) \\
& =1+(1+(1+(1+(1+(1+y))(1+x))))(1+x) \\
& =\neg(\neg y \oplus x) \oplus x, \\
& \neg(x \oplus \neg y) \oplus \neg(\neg x \oplus y) \\
& =1+(1+(1+(1+(1+x)(1+(1+y))))) \\
& \times(1+(1+(1+(1+(1+x))(1+y)))) \\
& =1+(1+(1+x) y)(1+x(1+y))=x+y
\end{aligned}
$$

and

$$
\neg(\neg x \oplus \neg y)=1+(1+(1+(1+x))(1+(1+y)))=x y
$$

for all $x, y, z \in A$.

\section{Strong De Morgan Algebras}

In this section we want to show that MV-algebras correspond in a natural bijective way to certain algebras that are similar to so-called De Morgan algebras. This yields also a natural bijective correspondence between these so-called strong De Morgan algebras and pseudorings.

Definition 4.1. A De Morgan algebra is an algebra $\left(A, \vee, \wedge,{ }^{\prime}, 0,1\right)$ of type $(2,2,1,0,0)$ such that $(A, \vee, \wedge, 0,1)$ is a bounded distributive lattice and ' is an antiendomorphism of $(A, \vee, \wedge)$. A strong De Morgan 
algebra is an algebra $\left(A, \vee, \wedge,\left({ }^{a} ; a \in A\right), 0,1\right)$ where $(A, \vee, \wedge, 0,1)$ is a bounded lattice, for each $a \in A,\left([a, 1], \vee, \wedge,{ }^{a}, a, 1\right)$ is a De Morgan algebra, $\left(x^{y}\right)^{y}=x$ for all $x, y \in A$ with $x \geq y$ and

$$
\left(x \vee(y \vee z)^{z}\right)^{(y \vee z)^{z}}=\left(y \vee(x \vee z)^{z}\right)^{(x \vee z)^{z}}
$$

for all $x, y, z \in A$.

Theorem 4.1. If $A$ is a set then the formulas

$$
\begin{aligned}
& x \vee y:=\neg(\neg x \oplus y) \oplus y, \\
& x \wedge y:=\neg(\neg(x \oplus \neg y) \oplus \neg y), \\
& x^{a}:=\neg x \oplus a
\end{aligned}
$$

and

$$
\begin{aligned}
& x \oplus y:=\left(x^{0} \vee y\right)^{y}, \\
& \neg x:=x^{0}
\end{aligned}
$$

for all $x, y, a \in A$ induce mutually inverse bijections between the set of all $M V$-algebras on $A$ and the set of all strong De Morgan algebras on A.

Proof. First let $(A, \oplus, \neg, 0)$ be an MV-algebra and put $x \vee y:=$ $\neg(\neg x \oplus y) \oplus y, x \wedge y:=\neg(\neg(x \oplus \neg y) \oplus \neg y)$ and $x^{a}:=\neg x \oplus a$ for all $x, y, a \in A$. It is well-known that $(A, \vee, \wedge, 0,1)$ is a bounded distributive lattice the corresponding partial order relation of which is given by $x \leq y$ if and only if $\neg x \oplus y=1(x, y \in A)$. Moreover, for $x, y, a \in A$

$$
\begin{aligned}
& a \leq x^{a}=\neg x \oplus a \quad \text { since } \neg a \oplus \neg x \oplus a=1, \\
& a \leq x \leq y \quad \text { implies } \neg(\neg y \oplus a) \oplus(\neg x \oplus a)=\neg(\neg y \oplus a) \oplus a \oplus \neg x \\
& \quad=\neg(\neg a \oplus y) \oplus y \oplus \neg x=1, \quad \text { i.e. } \quad y^{a} \leq x^{a},
\end{aligned}
$$

and

$a \leq x$ implies $\neg(\neg x \oplus a) \oplus a=\neg(\neg a \oplus x) \oplus x=x$, i.e. $\left(x^{a}\right)^{a}=x$.

Put $x \circ y:=(x \vee y)^{y}$ for all $x, y \in A$. Then

$x \circ y=\neg(\neg(\neg x \oplus y) \oplus y) \oplus y=\neg(\neg y \oplus \neg x \oplus y) \oplus \neg x \oplus y=\neg x \oplus y$

for all $x, y \in A$ and hence

$$
\begin{aligned}
& \left(x \vee(y \vee z)^{z}\right)^{(y \vee z)^{z}}=x \circ(y \circ z)=\neg x \oplus \neg y \oplus z=\neg y \oplus \neg x \oplus z \\
& \quad=y \circ(x \circ z)=\left(y \vee(x \vee z)^{z}\right)^{(x \vee z)^{z}}, \\
& \left(x^{0} \vee y\right)^{y}=\neg(\neg(\neg \neg x \oplus y) \oplus y) \oplus y=\neg(\neg y \oplus x \oplus y) \oplus x \oplus y=x \oplus y
\end{aligned}
$$


and

$$
x^{0}=\neg x \oplus 0=\neg x
$$

for all $x, y, z \in A$.

Conversely, if $\left(A, \vee, \wedge,\left({ }^{a} ; a \in A\right), 0,1\right)$ is a strong De Morgan algebra and $x \oplus y:=\left(x^{0} \vee y\right)^{y}, \neg x:=x^{0}$ and $x \circ y:=(x \vee y)^{y}$ for all $x, y \in A$ then

$$
\begin{aligned}
& x \circ(y \circ z)=y \circ(x \circ z), \\
& (x \circ 0) \circ 0=x^{00}=x, \\
& x \oplus y=(x \circ 0) \circ y, \\
& x \oplus y=(x \circ 0) \circ y=(x \circ 0) \circ((y \circ 0) \circ 0)=(y \circ 0) \circ((x \circ 0) \circ 0) \\
& \quad=(y \circ 0) \circ x=y \oplus x, \\
& (x \oplus y) \oplus z=z \oplus(x \oplus y)=(z \circ 0) \circ((x \circ 0) \circ y) \\
& \quad=(x \circ 0) \circ((z \circ 0) \circ y)=x \oplus(z \oplus y)=x \oplus(y \oplus z), \\
& \begin{aligned}
x \oplus 0=(x \circ 0) \circ 0=x, \\
x \oplus 1=\left(x^{0} \vee 1\right)^{1}=1, \\
\neg x \oplus y=\left(\left(x^{0}\right)^{0} \vee y\right)^{y}=(x \vee y)^{y}, \\
\neg(\neg x \oplus y) \oplus y=\left((x \vee y)^{y} \vee y\right)^{y}=\left((x \vee y)^{y}\right)^{y}=x \vee y
\end{aligned}
\end{aligned}
$$

and hence

$$
\begin{aligned}
& \neg(\neg x \oplus y) \oplus y=\neg(\neg y \oplus x) \oplus x, \\
& \neg(\neg(x \oplus \neg y) \oplus \neg y)=\neg(\neg(\neg \neg x \oplus \neg y) \oplus \neg y)=\left(x^{0} \vee y^{0}\right)^{0}=x \wedge y
\end{aligned}
$$

and

$$
\neg x \oplus a=(x \vee a)^{a}=x^{a} \quad \text { if } \quad x \geq a
$$

for all $x, y, z, a \in A$.

Combining Theorems 3.1 and 4.1 yields

Theorem 4.2. If $A$ is a set then the formulas

$$
\begin{aligned}
& x \vee y:=1+(1+x(1+y))(1+y), \\
& x \wedge y:=(1+(1+x) y) y, \\
& x^{a}:=1+x(1+a)
\end{aligned}
$$


and

$$
\begin{aligned}
& x+y:=\left(\left(x^{0} \vee y^{0}\right)^{y^{0}} \vee\left((x \vee y)^{y}\right)^{0}\right)^{\left((x \vee y)^{y}\right)^{0},} \\
& x y:=\left(\left(x \vee y^{0}\right)^{y^{0}}\right)^{0}
\end{aligned}
$$

for all $x, y, a \in A$ induce mutually inverse bijections between the set of all pseudorings on $A$ and the set of all strong De Morgan algebras on $A$.

As an immediate consequence of Theorems 3.1, 4.1 and 4.2 and their proofs we obtain

Corollary 4.1. If $(R,+, \cdot, 1)$ is a pseudoring and

$$
\left(R, \vee, \wedge,\left({ }^{a} ; a \in R\right), 0,1\right)
$$

denotes the corresponding strong De Morgan algebra then (i) and (ii) hold:

(i) $a \leq b$ if and only if $a(1+b)=0$

(ii) $\left(R, \vee, \wedge,{ }^{0}, 0,1\right)$ is a Boolean algebra if and only if $(1+x x) x=0$ for every $x \in R$.

\section{References}

[1] ChANG, C. C. (1958) Algebraic analysis of many valued logics. Trans. AMS 88: 467-490

[2] ChaJda, I. (1996) Pseudosemirings induced by ortholattices. Czechoslovak Math. J. 46: 405-411

[3] Chajda, I., Eigenthaler, G. (1998) A note on orthopseudorings and Boolean quasirings. Sitzungsber. Österr. Akad. Wiss. Math.-naturwiss. Kl., Abt. II 207: 83-94

[4] ChaJdA, I., LÄnger, H. (2000) Ring-like operations in pseudocomplemented semilattices. Discuss. Math. General Algebra Appl. 20: 87-95

[5] Chajda, I., LÄnger, H. (2004) Orthorings. Discuss. Math. General Algebra Appl. 24: 137-147

[6] Chajda, I., LÄNGER, H., MACZYŃSKI, M. (2004) Ring-like structures corresponding to generalized orthomodular lattices. Math. Slovaca 54: 143-150

[7] Cignoli, R. L. O., D’Ottaviano, I. M. L., Mundici, D. (2000) Algebraic Foundations of Many-valued Reasoning. Kluwer, Dordrecht

[8] Di Nola, A., Gerla, B. (2005) Algebras of Łukasiewicz's logic and their semiring reducts. Proc. Conf. Idempotent Math. Math. Phys. (Litvinov, G. L., Maslov, V. P., eds.). Contemporary Math., pp. 131-144. AMS, Providence, RI

[9] Dorfer, G., DVUREČENSKIJ, A., LÄNGER, H. (1996) Symmetric difference in orthomodular lattices. Math. Slovaca 46: 435-444

[10] DORninger, D., LÄNGER, H., MACZYŃSKI, M. (1997) The logic induced by a system of homomorphisms and its various algebraic characterizations. Demonstratio Math. 30: 215-232 
[11] DORNINGER, D., LÄNGER, H., MACZYŃSKI, M. (1997) On ring-like structures occurring in axiomatic quantum mechanics. Sitzungsber. Österr. Akad. Wiss. Math.-naturwiss. Kl., Abt. II 206: 279-289

[12] DORninger, D., LÄNGER, H., MACZYŃSKI, M. (1999) On ring-like structures induced by Mackey's probability function. Rep. Math. Phys. 43: 499-515

[13] DORNINGER, D., LÄNGER, H., MACZYŃSKI, M. (2000) Lattice properties of ringlike quantum logics. Intern. J. Theor. Phys. 39: 1015-1026

[14] Dorninger, D., LÄNGER, H., MACZYŃSKI, M. (2001) Concepts of measures on ring-like quantum logics. Rep. Math. Phys. 47: 167-176

[15] DORNINGER, D., LÄNGER, H., MACZYŃSKI, M. (2001) Ring-like structures with unique symmetric difference related to quantum logic. Discuss. Math. General Algebra Appl. 21: 239-253

[16] DvurečEnskiJ, A., Pulmannová, S. (2000) New Trends in Quantum Structures. Kluwer, Dordrecht, and Ister Science, Bratislava

[17] LÄNGER, H. (1998) Generalizations of the correspondence between Boolean algebras and Boolean rings to orthomodular lattices. Tatra Mt. Math. Publ. 15: 97-105

Authors' addresses: Ivan Chajda, Department of Algebra and Geometry, Palacký University Olomouc, Tomkova 40, 77900 Olomouc, Czech Republic, E-Mail: chajda@inf.upol.cz; Helmut Länger, Institute of Discrete Mathematics and Geometry, Research Unit Algebra, Vienna University of Technology, Wiedner Hauptstraße 8-10, 1040 Vienna, Austria, E-Mail: h.laenger@tuwien.ac.at 